Steffen Brasch · Katharina Habermann · Lutz Habermann

\title{
Symplectic Dirac operators on Hermitian symmetric spaces
}

Received: 20 November 2008 / Revised: 29 May 2009

Published online: 14 July 2009

(C) The Author(s) 2009. This article is published with open access at Springerlink.com

\begin{abstract}
We describe the shape of the symplectic Dirac operators on Hermitian symmetric spaces. For this, we consider these operators as families of operators that can be handled more easily than the original ones.
\end{abstract}

\section{Introduction}

Symplectic spinor fields were introduced by Kostant [16] in the early 1970s. This was done in the context of geometric quantization, where he was interested in constructing the so-called half-form bundle as well as the half-form pairing in order to establish the appropriate Hilbert space. Kostants construction was quite an essential step in geometric quantization, but afterwards no further work has been done to seek out interior aspects of symplectic spin geometry for two decades.

In 1995, the second author introduced symplectic Dirac operators and initialized a systematical investigation [8]. Some progress has been made in understanding basic properties of these operators [9]. Moreover, symplectic Dirac operators have been studied in the setting of parabolic geometries (cf. e.g. $[14,17])$ and received attention in mathematical physics (cf. e.g. [20]). A systematic and thorough introduction to the subject can be found in [12].

There are several topics of their own interest that are relevant to get a broader understanding of symplectic Dirac operators. For example, a deeper knowledge of the moduli space of symplectic structures may provide a way of analyzing how the symplectic Dirac operators are related to the choice of the symplectic structure of the underlying symplectic manifold. For considerations on moduli spaces of symplectic structures, see $[6,7,23]$. Furthermore, the symplectic Dirac operators are defined by means of a symplectic connection. It is well-known that in symplectic

This work was partially supported by the Deutsche Forschungsgemeinschaft (DFG).

S. Brasch: Department of Mathematics and Computer Science, University of Greifswald, Jahnstr. 15a, 17487 Greifswald, Germany. e-mail: sbrasch@uni-greifswald.de

K. Habermann: SUB Göttingen, Platz der Göttinger Sieben 1, 37073 Göttingen, Germany. e-mail: habermann@sub.uni-goettingen.de

L. Habermann $(\varangle)$ : Institute of Differential Geometry, University of Hannover, Welfengarten 1, 30167 Hannover, Germany. e-mail: habermann@math.uni-hannover.de

Mathematics Subject Classification (2000): 32M15, 53C35, 53D05, 58J50 
geometry there is no analog of the Levi-Civita connection. With the aim to get a deeper insight into the structure of the space of symplectic connections, in [13] an approach of a purely symplectic Yang-Mills theory is given.

In order to develop the symplectic Dirac operator picture, it is significant to have more explicit examples. In the present paper, we study symplectic Dirac operators in the case where the underlying symplectic manifold is a Hermitian symmetric space. This gives a class of examples which were not figured out so far. In the particular case of odd-dimensional complex projective spaces, the spectrum of an associated second order operator has been already computed [10,24]. However, no calculations are performed for the first order symplectic Dirac operators there. Here, we are going to study the shape of these operators itself. Our strategy is based on the idea of considering symplectic Dirac operators as families of operators acting on more suitable spaces than the section space of an infinite rank vector bundle.

The paper is organized as follows: the necessary concepts of symplectic Dirac operators in the setting of symmetric symplectic spaces are introduced in Sect. 2. For the general situation, we refer to [12]. In Sect. 3 we describe a splitting of the symplectic spinor bundle into subbundles of finite rank and apply the Frobenius reciprocity to decompose the corresponding section spaces in the case of a Hermitian symmetric space. Moreover, we explain how the symplectic Dirac operators built with respect to the canonical Hermitian connection behave in relation to these decompositions. The last section is devoted to a detailed discussion of the case $\mathbb{C} P^{1}$.

The example of $\mathbb{C} P^{1}$ has already been considered by the second author in an earlier paper, see [10]. But two new aspects are relevant in the present paper. First, as already mentioned above, here the symplectic Dirac operators itself are investigated, whereas [10] contains results only for the associated second order operator. Second, the calculations are done in the unified context of Hermitian symmetric spaces. In contrast to that, in [10] the example of $\mathbb{C} P^{1}$ is studied as a stand alone example with specialized computations.

\section{Preliminaries}

Let $G$ be a simply connected real Lie group, let $H$ be a closed and connected subgroup of $G$ and set $M$ to be the homogeneous space $G / H$. Let $\mathfrak{g}$ and $\mathfrak{h}$ denote the Lie algebras of $G$ and $H$, respectively. We suppose that there exists a subspace $\mathfrak{m} \subset \mathfrak{g}$ such that

$$
\mathfrak{g}=\mathfrak{h} \oplus \mathfrak{m}
$$

as well as

$$
[\mathfrak{h}, \mathfrak{m}] \subset \mathfrak{m} \text { and }[\mathfrak{m}, \mathfrak{m}] \subset \mathfrak{h} \text {. }
$$

Moreover, let $\omega_{0}$ be an ad(h)-invariant non-degenerate skew-symmetric bilinear form on $\mathfrak{m}$. Identifying the tangent space $T_{o} M$ to $M$ at the point $o=e H$ with $\mathfrak{m}, \omega_{0}$ induces a $G$-invariant almost symplectic structure $\omega$ on $M$. Here and in the sequel, $e$ denotes the unit element of $G$. Since the $\operatorname{ad}(\mathfrak{h})$-invariance of $\omega_{0}$ is equivalent to the trivial extension of $\omega_{0}$ to $\mathfrak{g}$ being a Chevalley 2-cocycle, $\omega$ is closed. 
Thus $\omega$ is even a symplectic structure on $M$. In this way, we have described $(M, \omega)$ as a simply connected symplectic symmetric space (cf. [2]).

The canonical projection $\pi: G \rightarrow M$ and the right action of $H$ on $G$ give $G$ the structure of an $H$-principal fiber bundle. Let $\kappa: H \rightarrow \mathrm{GL}(\mathfrak{m})$ be the isotropy representation of the homogeneous space $M$, i.e. the restriction of the adjoint representation of $G$ to $H$ acting on $\mathfrak{m}$. By assumption, $\kappa$ maps into the symplectic group $\operatorname{Sp}(\mathfrak{m})$ of the symplectic vector space $\left(\mathfrak{m}, \omega_{0}\right)$. We fix a symplectic basis $\left(X_{1}, \ldots, X_{2 n}\right)$ of $\left(\mathfrak{m}, \omega_{0}\right)$, i.e. a basis of $\mathfrak{m}$ such that

$$
\omega_{0}\left(X_{j}, X_{k}\right)=\omega_{0}\left(X_{n+j}, X_{n+k}\right)=0 \text { and } \omega_{0}\left(X_{j}, X_{n+k}\right)=\delta_{j k}
$$

for $j, k=1, \ldots, n$, and identify the symplectic frame bundle $R$ of $(M, \omega)$ with the $\operatorname{Sp}(\mathfrak{m})$-principal fiber bundle $G \times{ }_{\kappa} \operatorname{Sp}(\mathfrak{m})$ associated to $G$ with respect to $\kappa: H \rightarrow \mathrm{Sp}(\mathfrak{m})$ via

$$
[g, A] \in G \times{ }_{\kappa} \mathrm{Sp}(\mathfrak{m}) \mapsto\left(\mathrm{d} \pi\left(\mathrm{d} L_{g}\left(A X_{1}\right)\right), \ldots, \mathrm{d} \pi\left(\mathrm{d} L_{g}\left(A X_{2 n}\right)\right)\right) \in R .
$$

Let $\operatorname{Mp}(\mathfrak{m})$ be the connected double covering group of $\operatorname{Sp}(\mathfrak{m})$ and let $\rho: \operatorname{Mp}(\mathfrak{m}) \rightarrow$ $\operatorname{Sp}(\mathfrak{m})$ denote the covering homomorphism. If $\tilde{\kappa}: H \rightarrow \operatorname{Mp}(\mathfrak{m})$ is a lift of $\kappa$, i.e. a homomorphism such that $\rho \circ \tilde{\kappa}=\kappa$, then the $\operatorname{Mp}(\mathfrak{m})$-principal fiber bundle $P=G \times_{\tilde{\kappa}} \mathrm{Mp}(\mathfrak{m})$ together with the map $F_{P}: P \rightarrow R$ defined by $F_{P}([g, q])=$ $[g, \rho(q)]$ is a metaplectic structure of the symplectic manifold $(M, \omega)$.

Theorem 2.1. The mapping $\tilde{\kappa} \mapsto\left(P, F_{P}\right)$ described above induces a $1: 1$ correspondence between the lifts $\tilde{\kappa}$ of $\kappa$ and the isomorphism classes of metaplectic structures $\left(P, F_{P}\right)$ of the simply connected symplectic symmetric space $(M, \omega)$.

Proof. One proceeds as in the Riemannian case (cf. [1,4,19]).

According to the above, we identify the tangent bundle $T M$ of $M$ with the vector bundle $G \times{ }_{\kappa} \mathfrak{m}$ associated to $G$ with respect to $\kappa$ via

$$
[g, X] \in G \times_{\kappa} \mathfrak{m} \mapsto \mathrm{d} \pi\left(\mathrm{d} L_{g} X\right) \in T M .
$$

The space of smooth vector fields on $M$, i.e. the space $\Gamma(T M)$ of smooth sections of $T M$ is then the space of smooth maps $\xi: G \rightarrow \mathfrak{m}$ such that

$$
\xi(g h)=\kappa\left(h^{-1}\right) \xi(g)
$$

for $g \in G$ and $h \in H$ and the symplectic structure $\omega$ is given by

$$
\omega([g, X],[g, Y])=\omega_{0}(X, Y) .
$$

Consequently, the function $\omega\left(\xi_{1}, \xi_{2}\right) \in C^{\infty}(M)$ for $\xi_{1}, \xi_{2} \in \Gamma(T M)$ satisfies

$$
\omega\left(\xi_{1}, \xi_{2}\right)(\pi(g))=\omega_{0}\left(\xi_{1}(g), \xi_{2}(g)\right) .
$$

Let $\nabla$ be the $G$-invariant connection on $M$ defined by

$$
\nabla_{[g, X]} \xi=\left[g, \mathrm{~d} \xi\left(\mathrm{d} L_{g} X\right)\right]
$$

for $[g, X] \in T M$ and $\xi \in \Gamma(T M)$. Then

$$
\left(\nabla_{\xi_{1}} \xi_{2}\right)(g)=\mathrm{d} \xi_{2}\left(\mathrm{~d} L_{g} \xi_{1}(g)\right)
$$

for $\xi_{1}, \xi_{2} \in \Gamma(T M)$ and $g \in G$. 
Proposition 2.2. The connection $\nabla$ is symplectic, i.e. $\nabla \omega=0$.

Proof. Let $\xi, \xi_{1}, \xi_{2} \in \Gamma(T M)$. By means of

$$
\xi(u)(\pi(g))=\mathrm{d}(u \circ \pi)\left(\mathrm{d} L_{g} \xi(g)\right)
$$

for $u \in C^{\infty}(M)$ and Eqs. (2.2) and (2.3), we conclude

$$
\begin{aligned}
\xi\left(\omega\left(\xi_{1}, \xi_{2}\right)\right)(\pi(g)) & =\mathrm{d}\left(\omega_{0}\left(\xi_{1}, \xi_{2}\right)\right)\left(\mathrm{d} L_{g} \xi(g)\right) \\
& =\omega_{0}\left(\mathrm{~d} \xi_{1}\left(\mathrm{~d} L_{g} \xi(g)\right), \xi_{2}(g)\right)+\omega_{0}\left(\xi_{1}(g), \mathrm{d} \xi_{2}\left(\mathrm{~d} L_{g} \xi(g)\right)\right) \\
& =\omega_{0}\left(\left(\nabla_{\xi} \xi_{1}\right)(g), \xi_{2}(g)\right)+\omega_{0}\left(\xi_{1}(g),\left(\nabla_{\xi} \xi_{2}\right)(g)\right) \\
& =\omega\left(\nabla_{\xi} \xi_{1}, \xi_{2}\right)(\pi(g))+\omega\left(\xi_{1}, \nabla_{\xi} \xi_{2}\right)(\pi(g)) .
\end{aligned}
$$

Moreover, we have (cf. [15,18])

Proposition 2.3. The connection $\nabla$ is torsion free.

Thus $\nabla$ is a torsion-free symplectic connection on the almost symplectic manifold $(M, \omega)$. By a classical result of Tondeur (cf. [21]), this also implies that $(M, \omega)$ is symplectic. The question when $\nabla$ is a so-called connection of Ricci type was studied in [5]. In particular, it was shown that if $(M, \omega)$ is compact then $(M, \omega)$ is the complex projective space $\mathbb{C} P^{n}$.

Using the fixed symplectic basis $\left(X_{1}, \ldots, X_{2 n}\right)$ of $\left(\mathfrak{m}, \omega_{0}\right)$, we identify $\operatorname{Sp}(\mathfrak{m})$ and its double cover $\operatorname{Mp}(\mathfrak{m})$ with the symplectic $\operatorname{group} \operatorname{Sp}(n, \mathbb{R})$ and the metaplectic group $\operatorname{Mp}(n, \mathbb{R})$, respectively. Let $\boldsymbol{m}: \operatorname{Mp}(\mathfrak{m}) \rightarrow \mathrm{U}\left(L^{2}\left(\mathbb{R}^{n}\right)\right)$ be the metaplectic representation. Let $\tilde{\kappa}$ be a lift of $\kappa$ and let $\left(P, F_{P}\right)$ be the metaplectic structure of $(M, \omega)$ constructed from $\tilde{\kappa}$ as described above. The symplectic spinor bundle is then the Hilbert space bundle $Q=G \times_{\lambda} L^{2}\left(\mathbb{R}^{n}\right)$ associated to $G$ with respect to $\lambda=\boldsymbol{m} \circ \tilde{\kappa}: H \rightarrow \mathrm{U}\left(L^{2}\left(\mathbb{R}^{n}\right)\right)$. Accordingly, a symplectic spinor field, i.e. a smooth section of $Q$ is a smooth map $\varphi: G \rightarrow L^{2}\left(\mathbb{R}^{n}\right)$ such that

$$
\varphi(g h)=\lambda\left(h^{-1}\right) \varphi(g)
$$

for $g \in G$ and $h \in H$. Since the symplectic connection $\nabla$ on $M$ is induced by the $G$-invariant connection on the $H$-principal fiber bundle $G$ that corresponds to the decomposition (2.1), the same holds true for the spinor derivative on $Q$, also denoted by $\nabla$. Therefore,

$$
\left(\nabla_{\xi} \varphi\right)(g)=\mathrm{d} \varphi\left(\mathrm{d} L_{g} \xi(g)\right)
$$

for $\varphi \in \Gamma(Q)$ and $\xi \in \Gamma(T M)$.

According to our conventions, the symplectic Clifford multiplication is the homomorphism $\mu_{0}: \mathfrak{m} \otimes L^{2}\left(\mathbb{R}^{n}\right) \rightarrow L^{2}\left(\mathbb{R}^{n}\right)$ given by

$$
\mu_{0}\left(X_{j} \otimes f\right)(x)=\mathrm{i} x_{j} f(x) \text { and } \mu_{0}\left(X_{n+j} \otimes f\right)=\frac{\partial f}{\partial x^{j}}
$$


for $f \in L^{2}\left(\mathbb{R}^{n}\right), x=\left(x_{1}, \ldots, x_{n}\right) \in \mathbb{R}^{n}$ and $j=1, \ldots, n$, where the multiplication by $X \in \mathfrak{m}$ has to be seen as an unbounded operator on $L^{2}\left(\mathbb{R}^{n}\right)$. As usual, we shall write $X \cdot f$ for $\mu_{0}(X \otimes f)$. The multiplication $\mu_{0}$ induces a multiplication $\mu: T M \otimes Q \rightarrow Q$ by

$$
\mu([g, X] \otimes[g, f])=[g, X \cdot f] .
$$

The first symplectic Dirac operator is now defined as

$$
\mathcal{D}=\mu \circ\left(\bar{\omega} \otimes \operatorname{id}_{Q}\right) \circ \nabla: \Gamma(Q) \rightarrow \Gamma(Q),
$$

where $\bar{\omega}$ means the isomorphism $\bar{\omega}: T^{*} M \rightarrow T M$ generated by the symplectic structure $\omega$ on $M$.

Proposition 2.4. The symplectic Dirac operator $\mathcal{D}$ can be written as

$$
\mathcal{D}(\varphi)=\sum_{j=1}^{n} X_{j} \cdot X_{n+j}(\varphi)-\sum_{j=1}^{n} X_{n+j} \cdot X_{j}(\varphi)
$$

for $\varphi \in \Gamma(Q)$, where $X(\varphi)$ for $X \in \mathfrak{m}$ denotes the derivative of $\varphi$ in the direction of the left-invariant vector field determined by $X$, i.e.

$$
X(\varphi)(g)=\mathrm{d} \varphi\left(\mathrm{d} L_{g} X\right) .
$$

Proof. Let $\xi_{1}, \ldots, \xi_{2 n} \in \Gamma(T M)$ such that $\xi_{j}(e)=X_{j}$ for $j=1, \ldots, 2 n$. By the local expression for $D$ (cf. [12]) and Eq. (2.4), we then have

$$
\begin{aligned}
\mathcal{D}(\varphi)(e) & =\sum_{j=1}^{n} \xi_{j}(e) \cdot\left(\nabla_{\xi_{n+j}} \varphi\right)(e)-\sum_{j=1}^{n} \xi_{n+j}(e) \cdot\left(\nabla_{\xi_{j}} \varphi\right)(e) \\
& =\sum_{j=1}^{n} X_{j} \cdot \mathrm{d} \varphi\left(X_{n+j}\right)-\sum_{j=1}^{n} X_{n+j} \cdot \mathrm{d} \varphi\left(X_{j}\right) \\
& =\sum_{j=1}^{n} X_{j} \cdot X_{n+j}(\varphi)(e)-\sum_{j=1}^{n} X_{n+j} \cdot X_{j}(\varphi)(e) .
\end{aligned}
$$

Since both sides of Eq. (2.6) describe $G$-invariant operators, this proves the proposition.

To construct the second Dirac operator $\tilde{\mathcal{D}}$, we need an $\omega$-compatible almost complex structure $J$ on $M$. We assume that $J$ is $G$-invariant. That is, we suppose that the endomorphism $J: T M \rightarrow T M$ is given by

$$
J[g, X]=\left[g, J_{0} X\right]
$$

for $[g, X] \in T M$, where $J_{0}$ is an ad(h)-invariant complex structure on the vector space $\mathfrak{m}$ such that

$$
\mathbf{g}_{0}(X, Y)=\omega_{0}\left(X, J_{0} Y\right)
$$


for $X, Y \in \mathfrak{m}$ defines an inner product $\mathbf{g}_{0}$ on $\mathfrak{m}$. Then

$$
\omega_{0}\left(J_{0} X, J_{0} Y\right)=\omega_{0}(X, Y) \text { and } \mathbf{g}_{0}\left(J_{0} X, J_{0} Y\right)=\mathbf{g}_{0}(X, Y)
$$

for any $X, Y \in \mathfrak{m}$. Let $\mathbf{g}$ denote the $G$-invariant Riemannian metric on $M$ induced by $\mathbf{g}_{0}$. This means that

$$
\mathbf{g}([g, X],[g, Y])=\mathbf{g}_{0}(X, Y) .
$$

By Eq. (2.7),

$$
\omega\left(J \xi_{1}, J \xi_{2}\right)=\omega\left(\xi_{1}, \xi_{2}\right) \text { and } \mathbf{g}\left(J \xi_{1}, J \xi_{2}\right)=\mathbf{g}\left(\xi_{1}, \xi_{2}\right)
$$

for all $\xi_{1}, \xi_{2} \in \Gamma(T M)$. Furthermore, it follows that $(M, g, J)$ is a Hermitian symmetric space and that $\nabla$ is its Levi-Civita connection.

The second symplectic Dirac operator is defined as

$$
\tilde{\mathcal{D}}=\mu \circ\left(\overline{\mathbf{g}} \otimes \operatorname{id}_{Q}\right) \circ \nabla: \Gamma(Q) \rightarrow \Gamma(Q),
$$

where $\overline{\mathbf{g}}: T^{*} M \rightarrow T M$ is the identification by means of the Riemannian metric $\mathbf{g}$ on $M$. Analogously to the proof of Proposition 2.4, one can show

Proposition 2.5. Suppose that the basis $\left(X_{1}, \ldots, X_{2 n}\right)$ of $\mathfrak{m}$ is unitary, i.e., in addition to symplecticity, it satisfies $J_{0} X_{j}=X_{n+j}$ for $j=1, \ldots, n$. Then the symplectic Dirac operator $\tilde{\mathcal{D}}$ takes the form

$$
\tilde{\mathcal{D}}(\varphi)=\sum_{j=1}^{2 n} X_{j} \cdot X_{j}(\varphi)
$$

for $\varphi \in \Gamma(Q)$.

\section{Decompositions and invariant subspaces}

First we decompose the symplectic spinor bundle $Q$ into subbundles of finite rank. For this, let $U(\mathfrak{m})$ denote the unitary group of the Hermitian vector space $\left(\mathfrak{m}, \mathbf{g}_{0}, J_{0}\right)$ and set $\hat{U}(\mathfrak{m})=\rho^{-1}(\mathrm{U}(\mathfrak{m}))$, which is a connected double cover of $U(\mathfrak{m})$. The irreducible components of the restriction $\boldsymbol{u}: \hat{\mathrm{U}}(\mathfrak{m}) \rightarrow \mathrm{U}\left(L^{2}\left(\mathbb{R}^{n}\right)\right)$ of the metaplectic representation $\boldsymbol{m}$ to $\hat{\mathrm{U}}(\mathfrak{m})$ can be described as follows. Let $\mathbb{N}_{0}$ denote the set of nonnegative integers and let $\boldsymbol{h}_{\alpha} \in L^{2}\left(\mathbb{R}^{n}\right)$ for a multi-index $\alpha=\left(\alpha_{1}, \ldots, \alpha_{n}\right) \in \mathbb{N}_{0}^{n}$ be the Hermite function on $\mathbb{R}^{n}$ defined by

$$
\boldsymbol{h}_{\alpha}(x)=\boldsymbol{h}_{\alpha_{1}}\left(x_{1}\right) \ldots \boldsymbol{h}_{\alpha_{n}}\left(x_{n}\right) \text {. }
$$

Here, $\boldsymbol{h}_{l}$ for $l \in \mathbb{N}_{0}$ are the classical Hermite functions on $\mathbb{R}$, which are given by

$$
\boldsymbol{h}_{l}(t)=\mathrm{e}^{t^{2} / 2} \frac{\mathrm{d}^{l}}{\mathrm{~d} t^{l}}\left(\mathrm{e}^{-t^{2}}\right) .
$$


As is well known, the Hermite functions $\boldsymbol{h}_{\alpha}$ form a complete orthogonal system in $L^{2}\left(\mathbb{R}^{n}\right)$. Furthermore, for any $l \in \mathbb{N}_{0}$, the span $\mathfrak{W}_{l}$ of the functions $\boldsymbol{h}_{\alpha}$ with $\alpha_{1}+\cdots+\alpha_{n}=l$ is an irreducible $\boldsymbol{u}$-invariant subspace of $L^{2}\left(\mathbb{R}^{n}\right)$ (cf. [3]).

Since the complex structure $J_{0}$ on $\mathfrak{m}$ is assumed to be ad(h)-invariant, the homomorphism $\kappa$ maps into $U(\mathfrak{m})$ and, therefore, the lift $\tilde{\kappa}$ maps into $\hat{U}(\mathfrak{m})$. Hence the unitary representation $\lambda: H \rightarrow \mathrm{U}\left(L^{2}\left(\mathbb{R}^{n}\right)\right)$ can be written as $\lambda=\boldsymbol{u} \circ \tilde{\kappa}$. Let $\boldsymbol{u}_{l}: \hat{\mathrm{U}}(\mathfrak{m}) \rightarrow \mathrm{U}\left(\mathfrak{W}_{l}\right)$ be the restriction of $\boldsymbol{u}$ to the subspace $\mathfrak{W}_{l}$ and set $\lambda_{l}=\boldsymbol{u}_{l} \circ \tilde{\kappa}$.

The above yields

Proposition 3.1. The symplectic spinor bundle $Q$ splits into the orthogonal sum of the finite rank subbundles $Q_{l}=G \times{ }_{\lambda_{l}} \mathfrak{W}_{l}, l \in \mathbb{N}_{0}$.

In the following considerations, we want to make use of the Frobenius reciprocity. To be able to do this, we assume from now on that $G$ is compact. Let $\tau_{\mathrm{a}}: G \rightarrow \mathrm{GL}\left(\mathfrak{V}_{\mathrm{a}}\right), \mathrm{a} \in \mathrm{A}$, form a complete system of representatives of isomorphism classes of irreducible complex representations of $G$. Let $v: H \rightarrow$ GL(W) be a finite-dimensional representation of $H$ and let $\operatorname{Hom}_{H}\left(\mathfrak{V}_{\mathrm{a}}, \mathfrak{W}\right)$ be the space of all $H$-equivariant homomorphisms $L: \mathfrak{V}_{\mathrm{a}} \rightarrow \mathfrak{W}$. We embed $\mathfrak{V}_{\mathrm{a}} \otimes \operatorname{Hom}_{H}\left(\mathfrak{V}_{\mathrm{a}}, \mathfrak{W}\right)$ into the space $\Gamma\left(G \times{ }_{v} \mathfrak{W}\right)$ of smooth sections of the associated vector bundle $G \times{ }_{v} \mathfrak{W}$ by assigning to $\mathrm{V} \otimes L \in \mathfrak{V}_{\mathrm{a}} \otimes \operatorname{Hom}_{H}\left(\mathfrak{V}_{\mathrm{a}}, \mathfrak{W}\right)$ the $H$-equivariant map

$$
g \in G \mapsto L\left(\tau_{\mathrm{a}}\left(g^{-1}\right) \mathrm{v}\right) \in \mathfrak{W} .
$$

For a proof of the theorem below, we refer to [22].

Theorem 3.2. (Frobenius reciprocity) The space $\Gamma\left(G \times{ }_{v} \mathfrak{W}\right)$ decomposes into the direct sum

$$
\sum_{\mathrm{a} \in \mathrm{A}} \mathfrak{V}_{\mathrm{a}} \otimes \operatorname{Hom}_{H}\left(\mathfrak{V}_{\mathrm{a}}, \mathfrak{W}\right) .
$$

Applying Theorem 3.2 to the representation $\boldsymbol{u}_{l}$, we obtain a decomposition of $\Gamma\left(Q_{l}\right)$ into the sum

$$
\sum_{\mathrm{a} \in \mathrm{A}} \mathfrak{V}_{\mathrm{a}} \otimes \operatorname{Hom}_{H}\left(\mathfrak{V}_{\mathrm{a}}, \mathfrak{W}_{l}\right) .
$$

Using this and Proposition 3.1, we now want to find invariant subspaces for the symplectic Dirac operators $D$ and $\tilde{D}$. We start with expressing the symplectic Clifford multiplication by means of the Hermite functions $\boldsymbol{h}_{\alpha}$.

Lemma 3.3. For any $\alpha \in \mathbb{N}_{0}^{n}$ and $j=1, \ldots, n$,

$$
X_{j} \cdot \boldsymbol{h}_{\alpha}=-\mathrm{i} \alpha_{j} \boldsymbol{h}_{\alpha-\langle j\rangle}-\frac{\mathrm{i}}{2} \boldsymbol{h}_{\alpha+\langle j\rangle}
$$

and

$$
X_{n+j} \cdot \boldsymbol{h}_{\alpha}=-\alpha_{j} \boldsymbol{h}_{\alpha-\langle j\rangle}+\frac{1}{2} \boldsymbol{h}_{\alpha+\langle j\rangle},
$$

where $\langle j\rangle=\left(\delta_{1 j}, \ldots, \delta_{n j}\right)$. 
Proof. This follows from Eqs. (2.5) and (3.1) and the relations

$$
\boldsymbol{h}_{l}^{\prime}(t)-t \boldsymbol{h}_{l}(t)=\boldsymbol{h}_{l+1}(t) \text { and } \boldsymbol{h}_{l}^{\prime}(t)+t \boldsymbol{h}_{l}(t)=-2 l \boldsymbol{h}_{l-1}(t)
$$

for the classical Hermite functions $\boldsymbol{h}_{l}$.

Lemma 3.4. Let $L \in \operatorname{Hom}_{H}\left(\mathfrak{V}_{\mathrm{a}}, \mathfrak{W}_{l}\right)$ and set

$$
\mathcal{D}_{\mathrm{a}}(L)=-\sum_{j=1}^{n} X_{j} \cdot L \circ\left(\tau_{\mathrm{a}}\right)_{*}\left(X_{n+j}\right)+\sum_{j=1}^{n} X_{n+j} \cdot L \circ\left(\tau_{\mathrm{a}}\right)_{*}\left(X_{j}\right) .
$$

Then

$$
\mathcal{D}_{\mathrm{a}}(L) \in \operatorname{Hom}_{H}\left(\mathfrak{V}_{\mathrm{a}}, \mathfrak{W}_{l-1}\right) \oplus \operatorname{Hom}_{H}\left(\mathfrak{V}_{\mathrm{a}}, \mathfrak{W}_{l+1}\right)
$$

with the convention that $\mathfrak{W}_{-1}=0$.

Proof. By Lemma 3.3,

$$
X \cdot \mathfrak{W}_{l} \subset \mathfrak{W}_{l-1} \oplus \mathfrak{W}_{l+1}
$$

for $X \in \mathfrak{m}$. Consequently, $\mathcal{D}_{\mathrm{a}}(L)$ is a homomorphism from $\mathfrak{V}_{\mathrm{a}}$ to $\mathfrak{W}_{l-1} \oplus \mathfrak{W}_{l+1}$. It remains to show that $\mathcal{D}_{\mathrm{a}}(L)$ is $H$-equivariant. Let $h \in H$ and $X, Y \in \mathfrak{m}$. Then

$$
\begin{aligned}
\tau_{\mathrm{a}}\left(h^{-1}\right) \circ\left(\tau_{\mathrm{a}}\right)_{*}(X) \circ \tau_{\mathrm{a}}(h) & =\left.\frac{\mathrm{d}}{\mathrm{d} t} \tau_{\mathrm{a}}\left(h^{-1}\right) \circ \tau_{\mathrm{a}}(\exp (t X)) \circ \tau_{\mathrm{a}}(h)\right|_{t=0} \\
& =\left.\tau_{\mathrm{a}}\left(\exp \left(t \operatorname{Ad}\left(h^{-1}\right) X\right)\right)\right|_{t=0} \\
& =\left(\tau_{\mathrm{a}}\right)_{*}\left(\kappa\left(h^{-1}\right) X\right) .
\end{aligned}
$$

Hence

$$
\left(\tau_{\mathrm{a}}\right)_{*}(X) \circ \tau_{\mathrm{a}}(h)=\tau_{\mathrm{a}}(h) \circ\left(\tau_{\mathrm{a}}\right)_{*}\left(\kappa\left(h^{-1}\right) X\right) .
$$

Since the symplectic Clifford multiplication is $\operatorname{Mp}(\mathfrak{m})$-equivariant, i.e.

$$
\rho(a) X \cdot \boldsymbol{m}(a) f=\boldsymbol{m}(a)(X \cdot f)
$$

for $a \in \operatorname{Mp}(\mathfrak{m})$ and $f \in L^{2}\left(\mathbb{R}^{n}\right)$, we furthermore have

$$
X \cdot \lambda(h) f=\lambda(h)\left(\kappa\left(h^{-1}\right) X \cdot f\right) .
$$

Equations (3.3) and (3.4) imply

$$
\begin{aligned}
X \cdot L \circ\left(\tau_{\mathrm{a}}\right)_{*}(Y) \circ \tau_{\mathrm{a}}(h) & =X \cdot L \circ \tau_{\mathrm{a}}(h) \circ\left(\tau_{\mathrm{a}}\right)_{*}\left(\kappa\left(h^{-1}\right) Y\right) \\
& =X \cdot \lambda(h) \circ L \circ\left(\tau_{\mathrm{a}}\right)_{*}\left(\kappa\left(h^{-1}\right) Y\right) \\
& =\lambda(h) \circ\left(\kappa\left(h^{-1}\right) X \cdot L \circ\left(\tau_{\mathrm{a}}\right)_{*}\left(\kappa\left(h^{-1}\right) Y\right)\right) .
\end{aligned}
$$

Since the definition of $\mathcal{D}_{\mathrm{a}}(L)$ does not depend on the choice of the symplectic basis $\left(X_{1}, \ldots, X_{2 n}\right)$ and since $\left(\kappa(h) X_{1}, \ldots, \kappa(h) X_{2 n}\right)$ for any $h \in H$ is again a symplectic basis, this proves the lemma. 
For $a \in A$, set

$$
\mathbf{U}_{\mathrm{a}}=\sum_{l=0}^{\infty} \operatorname{Hom}_{H}\left(\mathfrak{V}_{\mathrm{a}}, \mathfrak{W}_{l}\right) .
$$

By Lemma 3.4, Eq. (3.2) defines a homomorphism $\mathcal{D}_{\mathrm{a}}: \mathbf{U}_{\mathrm{a}} \rightarrow \mathbf{U}_{\mathrm{a}}$. The symplectic Dirac operator $\mathcal{D}$ and the homomorphisms $\mathcal{D}_{\mathrm{a}}$ are related by

Lemma 3.5. If $\mathbf{v} \otimes L \in \mathfrak{V}_{\mathrm{a}} \otimes \mathbf{U}_{\mathrm{a}}$, then

$$
\mathcal{D}(\mathrm{v} \otimes L)=\mathrm{v} \otimes \mathcal{D}_{\mathrm{a}}(L) .
$$

Proof. We deduce that

$$
\begin{aligned}
X(\mathrm{v} \otimes L)(g) & =\left.\frac{\mathrm{d}}{\mathrm{d} t}(\mathrm{v} \otimes L)(g \exp (t X))\right|_{t=0} \\
& =\left.\frac{\mathrm{d}}{\mathrm{d} t} L\left(\tau_{\mathrm{a}}\left(\exp (-t X) g^{-1}\right) \mathrm{v}\right)\right|_{t=0} \\
& =-L \circ\left(\tau_{\mathrm{a}}\right)_{*}(X)\left(\tau_{\mathrm{a}}\left(g^{-1}\right) \mathrm{v}\right)
\end{aligned}
$$

for $X \in \mathfrak{m}$ and $g \in G$. This together with Proposition 2.4 gives the assertion.

The decompositions obtained above and Lemma 3.5 allow us to consider the symplectic Dirac operator $\mathcal{D}$ as a map

$$
\mathcal{D}: \sum_{\mathrm{a} \in \mathrm{A}} \mathfrak{V}_{\mathrm{a}} \otimes \mathbf{U}_{\mathrm{a}} \rightarrow \sum_{\mathrm{a} \in \mathrm{A}} \mathfrak{V}_{\mathrm{a}} \otimes \mathbf{U}_{\mathrm{a}} .
$$

Moreover, we have

Proposition 3.6. For any $\mathrm{a} \in \mathrm{A}$, the space $\mathfrak{V}_{\mathrm{a}} \otimes \mathbf{U}_{\mathrm{a}}$ is invariant under $\mathcal{D}$.

Proof. This is a consequence of Lemmas 3.4 and 3.5.

The same can be done for the symplectic Dirac operator $\tilde{\mathcal{D}}$ and the second order operator $\mathcal{P}=\mathrm{i}[\tilde{\mathcal{D}}, \mathcal{D}]$.

Lemma 3.7. Let $L \in \operatorname{Hom}_{H}\left(\mathfrak{V}_{\mathrm{a}}, \mathfrak{W}_{l}\right)$ and set

$$
\tilde{\mathcal{D}}_{\mathrm{a}}(L)=-\sum_{j=1}^{2 n} X_{j} \cdot L \circ\left(\tau_{\mathrm{a}}\right)_{*}\left(X_{j}\right),
$$

where here the basis $\left(X_{1}, \ldots, X_{2 n}\right)$ of $\mathfrak{m}$ is assumed to be unitary. Then

$$
\tilde{\mathcal{D}}_{\mathrm{a}}(L) \in \operatorname{Hom}_{H}\left(\mathfrak{V}_{\mathrm{a}}, \mathfrak{W}_{l-1}\right) \oplus \operatorname{Hom}_{H}\left(\mathfrak{V}_{\mathrm{a}}, \mathfrak{W}_{l+1}\right) .
$$

Proof. Using that $\kappa(h)$ is unitary for all $h \in H$, the proof is the same as the proof of Lemma 3.4.

Consequently, Eq. (3.5) gives rise to a homomorphism $\tilde{\mathcal{D}}_{\mathrm{a}}: \mathbf{U}_{\mathrm{a}} \rightarrow \mathbf{U}_{\mathrm{a}}$. 
Lemma 3.8. Let $\mathrm{v} \otimes L \in \mathfrak{V}_{\mathrm{a}} \otimes \mathbf{U}_{\mathrm{a}}$. Then

$$
\tilde{\mathcal{D}}(\mathrm{v} \otimes L)=\mathrm{v} \otimes \tilde{\mathcal{D}}_{\mathrm{a}}(L) \text { and } \mathcal{P}(\mathrm{v} \otimes L)=\mathrm{v} \otimes \mathcal{P}_{\mathrm{a}}(L),
$$

where $\mathcal{P}_{\mathrm{a}}=\mathrm{i}\left[\tilde{\mathcal{D}}_{\mathrm{a}}, \mathcal{D}_{\mathrm{a}}\right]$.

Proof. This follows from Proposition 2.5 and Lemma 3.5.

Proposition 3.9. The spaces $\mathfrak{V}_{\mathrm{a}} \otimes \mathbf{U}_{\mathrm{a}}$ are invariant under $\tilde{\mathcal{D}}$ and $\mathcal{P}$.

Proof. This is immediate from Lemmas 3.7 and 3.8 and Proposition 3.6.

Example 3.10. We consider the complex projective space $\mathbb{C} P^{n}=\mathrm{SU}(n+1) / \mathrm{U}(n)$, where the group $\mathrm{U}(n)$ is embedded into $\mathrm{SU}(n+1)$ by means of

$$
B \in \mathrm{U}(n) \mapsto\left(\begin{array}{ll}
\operatorname{det}(B)^{-1} & 0 \\
0 & B
\end{array}\right) \in \mathrm{SU}(n+1) .
$$

Let $\mathfrak{s u}(n+1)$ and $\mathfrak{u}(n)$ denote the Lie algebras of $\mathrm{SU}(n+1)$ and $\mathrm{U}(n)$, respectively, and let $\mathfrak{m}$ be the image of the homomorphism

$$
\Phi: z \in \mathbb{C}^{n} \mapsto\left(\begin{array}{ll}
0 & -\bar{z}^{\mathrm{T}} \\
z & 0
\end{array}\right) \in \mathfrak{s u}(n+1) .
$$

Then $\mathfrak{m}$ is an $\operatorname{ad}(\mathfrak{u}(n))$-invariant complement of $\mathfrak{u}(n) \subset \mathfrak{s u}(n+1)$ satisfying $[\mathfrak{m}, \mathfrak{m}] \subset \mathfrak{u}(n)$. We identify $\mathfrak{m}$ with $\mathbb{C}^{n}$ via $\Phi$. With this, the isotropy representation $\kappa$ takes the form

$$
\kappa(B)=\operatorname{det}(B) B
$$

for $B \in \mathrm{U}(n)$. Moreover, $\omega_{0}, J_{0}$ and $\mathbf{g}_{0}$ are the standard symplectic form, complex structure and inner product on $\mathbb{C}^{n}$, i.e.

$$
\omega_{0}(z, w)=\operatorname{Im}\left(\bar{z}^{\mathrm{T}} w\right), \quad J_{0} z=\mathrm{i} z \quad \text { and } \quad \mathbf{g}_{0}(z, w)=\operatorname{Re}\left(\bar{z}^{\mathrm{T}} w\right)
$$

for $z, w \in \mathbb{C}^{n}$. There exists a lift $\tilde{\kappa}$ of $\kappa$ iff $n$ is odd and in this case it is unique (for more details, see [24]). Since $\kappa$ maps onto $\mathrm{U}(n)$ and since the representations $\boldsymbol{u}_{l}$ are irreducible and pairwise inequivalent, by Schur's lemma, $\operatorname{Hom}_{H}\left(\mathfrak{V}_{\mathrm{a}}, \mathfrak{W}_{l}\right)$ is non-trivial for only finitely many $a \in A$. Therefore, the spaces $\mathfrak{V}_{\mathrm{a}} \otimes \mathbf{U}_{\mathrm{a}}$ have finite dimension.

In the next section, we examine the case of the complex projective line $\mathbb{C} P^{1}=$ $\mathrm{SU}(2) / \mathrm{U}(1)$ in more detail. 


\section{Symplectic Dirac operators on $\mathbb{C} P^{1}$}

We set

$$
E_{0}=\left(\begin{array}{ll}
\mathrm{i} & 0 \\
0 & \mathrm{i}
\end{array}\right), \quad E_{1}=\left(\begin{array}{ll}
0 & 1 \\
-1 & 0
\end{array}\right), \quad E_{2}=\left(\begin{array}{ll}
0 & \mathrm{i} \\
\mathrm{i} & 0
\end{array}\right) .
$$

The matrices $E_{0}, E_{1}, E_{2}$ form a basis of the Lie algebra $\mathfrak{s u}(2)$ and satisfy

$$
\left[E_{0}, E_{1}\right]=2 E_{2}, \quad\left[E_{0}, E_{2}\right]=-2 E_{1}, \quad\left[E_{1}, E_{2}\right]=2 E_{0} .
$$

Furthermore, the subalgebra $\mathfrak{u}(1) \subset \mathfrak{s u}(2)$ is spanned by $E_{0}$ and $\left\{E_{1}, E_{2}\right\}$ is a symplectic basis of the complement $\mathfrak{m}$. Since the complex structure $J_{0}$ on $\mathfrak{m}$ is given by $J_{0} E_{1}=E_{2}$, this basis is also unitary. We set $J_{0}=\rho_{*}^{-1}\left(J_{0}\right)$ and define a homomorphism $\tilde{\kappa}: \mathrm{U}(1) \rightarrow \hat{\mathrm{U}}(\mathfrak{m})$ by

$$
\tilde{\kappa}_{*}\left(E_{0}\right)=2 \jmath 0 .
$$

By Eq. (4.1),

$$
\kappa_{*}\left(E_{0}\right)=\left(\begin{array}{ll}
0 & -2 \\
2 & 0
\end{array}\right)=2 J_{0}
$$

Consequently, $\rho_{*} \circ \tilde{\kappa}_{*}=\kappa_{*}$, which implies $\rho \circ \tilde{\kappa}=\kappa$. Hence $\tilde{\kappa}$ is a lift of $\kappa$.

Let $\mathfrak{V}_{k}$ for $k \in \mathbb{N}_{0}$ be the vector space of homogeneous polynomials of degree $k$ in two variables $z_{1}$ and $z_{2}$ with complex coefficients. The polynomials $p_{k, 0}, \ldots, p_{k, k}$ defined by

$$
p_{k, j}\left(z_{1}, z_{2}\right)=z_{1}^{k-j} z_{2}^{j}
$$

form a basis of $\mathfrak{V}_{k}$. Let $\tau_{k}: \mathrm{SU}(2) \rightarrow \mathrm{GL}\left(\mathfrak{V}_{k}\right)$ be the representation of $\mathrm{SU}(2)$ induced by the canonical action of $\mathrm{SU}(2)$ on $\mathbb{C}^{2}$, i.e.

$$
\left(\tau_{k}(g) p\right)(z)=p\left(g^{-1} z\right)
$$

for $g \in \mathrm{SU}(2), p \in \mathfrak{V}_{k}$ and $z \in \mathbb{C}^{2}$. As it is well-known (cf. e.g. [25]), the representations $\tau_{k}$ are irreducible and pairwise inequivalent and these are all irreducible complex representations of SU(2) up to isomorphism. Moreover, one has

Lemma 4.1. For all $k \in \mathbb{N}_{0}$ and $j=0, \ldots, k$,

$$
\begin{aligned}
& \left(\tau_{k}\right)_{*}\left(E_{0}\right) p_{k, j}=\mathrm{i}(2 j-k) p_{k, j}, \\
& \left(\tau_{k}\right)_{*}\left(E_{1}\right) p_{k, j}=(j-k) p_{k, j+1}+j p_{k, j-1}, \\
& \left(\tau_{k}\right)_{*}\left(E_{2}\right) p_{k, j}=\mathrm{i}(j-k) p_{k, j+1}-\mathrm{i} j p_{k, j-1} .
\end{aligned}
$$

Lemma 4.2. Let $k, l \in \mathbb{N}_{0}$. If $(k+1) / 2+l \in\{0, \ldots, k\}$, then the space $\operatorname{Hom}_{\mathrm{U}(1)}\left(\mathfrak{V}_{k}, \mathfrak{W}_{l}\right)$ is one-dimensional and generated by the linear map $L_{k, l}$ : $\mathfrak{V}_{k} \rightarrow \mathfrak{W}_{l}$ defined by

$$
L_{k, l}\left(p_{k, j}\right)=\left\{\begin{array}{ll}
\boldsymbol{h}_{l} & \text { for } j=(k+1) / 2+l \\
0 & \text { else }
\end{array} .\right.
$$

In all other cases, $\operatorname{Hom}_{\mathrm{U}(1)}\left(\mathfrak{V}_{k}, \mathfrak{W}_{l}\right)$ is trivial. 
Proof. Let $\mathrm{H}_{0}: L^{2}(\mathbb{R}) \rightarrow L^{2}(\mathbb{R})$ be the Hamilton operator of the one-dimensional harmonic oscillator, i.e.

$$
\left(\mathrm{H}_{0} f\right)(x)=\frac{1}{2}\left(\frac{\mathrm{d}^{2} f}{\mathrm{~d} x^{2}}(x)-x^{2} f(x)\right) .
$$

Then (cf. [12])

$$
\boldsymbol{m}_{*}\left(J_{0}\right)=-\mathrm{iH}
$$

and

$$
\mathrm{H}_{0} \boldsymbol{h}_{l}=-\frac{2 l+1}{2} \boldsymbol{h}_{l} .
$$

Hence

$$
\left(\boldsymbol{u}_{l} \circ \tilde{\kappa}\right)_{*}\left(E_{0}\right) \boldsymbol{h}_{l}=2 \boldsymbol{m}_{*}\left(J_{0}\right) \boldsymbol{h}_{l}=-2 \mathrm{iH}_{0} \boldsymbol{h}_{l}=\mathrm{i}(2 l+1) \boldsymbol{h}_{l} .
$$

Applying Schur's lemma, the assertion now follows from Lemma4.1.

As shown in the previous section, the spaces $\mathfrak{V}_{k} \otimes \mathbf{U}_{k}$ with

$$
\mathbf{U}_{k}=\sum_{l=0}^{\infty} \operatorname{Hom}_{\mathrm{U}(1)}\left(\mathfrak{V}_{k}, \mathfrak{W}_{l}\right)
$$

are invariant under the symplectic Dirac operators $\mathcal{D}$ and $\tilde{\mathcal{D}}$.

Corollary 4.3. If $k$ is odd, then

$$
\mathbf{U}_{k}=\sum_{l=0}^{(k-1) / 2} \operatorname{Hom}_{\mathrm{U}(1)}\left(\mathfrak{V}_{k}, \mathfrak{W}_{l}\right)
$$

and $\operatorname{dim}\left(\mathfrak{V}_{k} \otimes \mathbf{U}_{k}\right)=(k+1)^{2} / 2$. If $k$ is even, then $\mathbf{U}_{k}$ is trivial.

Proof. This is a consequence of Lemma 4.2 and $\operatorname{dim} \mathfrak{V}_{k}=k+1$.

According to Lemmas 3.5 and 3.8, in order to calculate the symplectic Dirac operators $\mathcal{D}$ and $\tilde{\mathcal{D}}$ on $\mathbb{C} P^{1}$, we have to compute the linear maps $\mathcal{D}_{k}, \tilde{\mathcal{D}}_{k}: \mathbf{U}_{k} \rightarrow \mathbf{U}_{k}$ given by

$$
\mathcal{D}_{k}(L)=-E_{1} \cdot L \circ\left(\tau_{k}\right)_{*}\left(E_{2}\right)+E_{2} \cdot L \circ\left(\tau_{k}\right)_{*}\left(E_{1}\right)
$$

and

$$
\tilde{\mathcal{D}}_{k}(L)=-E_{1} \cdot L \circ\left(\tau_{k}\right)_{*}\left(E_{1}\right)-E_{2} \cdot L \circ\left(\tau_{k}\right)_{*}\left(E_{2}\right) .
$$

Thereby, we may assume that $k$ is odd. Then the maps $L_{k, 0}, \ldots, L_{k,(k-1) / 2}$ defined in Lemma 4.2 form a basis of $\mathbf{U}_{k}$. 
Proposition 4.4. For $l=0, \ldots,(k-1) / 2$,

$$
\mathcal{D}_{k}\left(L_{k, l}\right)=l(k+1-2 l) L_{k, l-1}+\left(\frac{k+1}{2}+l+1\right) L_{k, l+1}
$$

and

$$
\tilde{\mathcal{D}}_{k}\left(L_{k, l}\right)=-\mathrm{i} l(k+1-2 l) L_{k, l-1}+\mathrm{i}\left(\frac{k+1}{2}+l+1\right) L_{k, l+1} .
$$

Proof. Assume that $j=(k+1) / 2+l$. Then, by Lemmas 3.3 and 4.1, we get

$$
\begin{aligned}
\mathcal{D}_{k}\left(L_{k, l}\right)\left(p_{k, j-1}\right)= & -E_{1} \cdot L_{k, l}\left(\left(\tau_{k}\right)_{*}\left(E_{2}\right) p_{k, j-1}\right) \\
& +E_{2} \cdot L_{k, l}\left(\left(\tau_{k}\right)_{*}\left(E_{1}\right) p_{k, j-1}\right) \\
= & -E_{1} \cdot L_{k, l}\left(\mathrm{i}(j-k-1) p_{k, j}-\mathrm{i}(j-1) p_{k, j-2}\right) \\
& +E_{2} \cdot L_{k, l}\left((j-k-1) p_{k, j}+(j-1) p_{k, j-2}\right) \\
= & (j-k-1)\left(-\mathrm{i} E_{1} \cdot \boldsymbol{h}_{l}+E_{2} \cdot \boldsymbol{h}_{l}\right) \\
= & -2 l(j-k-1) \boldsymbol{h}_{l-1} \\
= & l(k+1-2 l) \boldsymbol{h}_{l-1}
\end{aligned}
$$

and

$$
\begin{aligned}
\mathcal{D}_{k}\left(L_{k, l}\right)\left(p_{k, j+1}\right)= & -E_{1} \cdot L_{k, l}\left(\left(\tau_{k}\right)_{*}\left(E_{2}\right) p_{k, j+1}\right) \\
& +E_{2} \cdot L_{k, l}\left(\left(\tau_{k}\right)_{*}\left(E_{1}\right) p_{k, j+1}\right) \\
= & -E_{1} \cdot L_{k, l}\left(\mathrm{i}(j-k+1) p_{k, j+2}-\mathrm{i}(j+1) p_{k, j}\right) \\
& +E_{2} \cdot L_{k, l}\left((j-k+1) p_{k, j+2}+(j+1) p_{k, j}\right) \\
= & (j+1)\left(\mathrm{i} E_{1} \cdot \boldsymbol{h}_{l}+E_{2} \cdot \boldsymbol{h}_{l}\right) \\
= & (j+1) \boldsymbol{h}_{l+1} \\
= & \left(\frac{k+1}{2}+l+1\right) \boldsymbol{h}_{l+1} .
\end{aligned}
$$

Analogously, we see

$$
\tilde{\mathcal{D}}_{k}\left(L_{k, l}\right)\left(p_{k, j-1}\right)=-\mathrm{i} l(k+1-2 l) \boldsymbol{h}_{l-1}
$$

and

$$
\tilde{\mathcal{D}}_{k}\left(L_{k, l}\right)\left(p_{k, j+1}\right)=\mathrm{i}\left(\frac{k+1}{2}+l+1\right) \boldsymbol{h}_{l+1} .
$$

This together with Lemmas 3.4 and 3.7 yields the claim.

We now normalize the homomorphisms $L_{k, 0}, \ldots, L_{k,(k-1) / 2}$ by

$$
L_{k, l}^{\circ}:=\sqrt{\frac{((k+1) / 2+l) !((k-1) / 2-l) !}{2^{l} l !}} L_{k, l}
$$

and set

$$
a_{k, l}:=\sqrt{2 l\left(\frac{(k+1)^{2}}{4}-l^{2}\right)} .
$$

Then, a straightforward calculation gives 
Corollary 4.5. For $l=0, \ldots,(k-1) / 2$,

$$
\mathcal{D}_{k}\left(L_{k, l}^{\circ}\right)=a_{k, l} L_{k, l-1}^{\circ}+a_{k, l+1} L_{k, l+1}^{\circ}
$$

and

$$
\tilde{\mathcal{D}}_{k}\left(L_{k, l}^{\circ}\right)=-\mathrm{i} a_{k, l} L_{k, l-1}^{\circ}+\mathrm{i} a_{k, l+1} L_{k, l+1}^{\circ} .
$$

In particular, the matrix representations of $\mathcal{D}_{k}$ and $\tilde{\mathcal{D}}_{k}$ with respect to the basis $\left\{L_{k, 0}^{\circ}, \ldots, L_{k,(k-1) / 2}^{\circ}\right\}$ are Hermitian.

We draw the following conclusions from Corollary 4.5. First, for the operators $\mathcal{P}_{k}=\mathrm{i}\left[\tilde{\mathcal{D}}_{k}, \mathcal{D}_{k}\right]$, we obtain

Corollary 4.6. For $l=0, \ldots,(k-1) / 2$,

$$
\begin{aligned}
\mathcal{P}_{k}\left(L_{k, l}^{\circ}\right) & =2\left(a_{k, l+1}^{2}-a_{k, l}^{2}\right) L_{k, l}^{\circ} \\
& =\left((k+1)^{2}-3(2 l+1)^{2}-1\right) L_{k, l}^{\circ} .
\end{aligned}
$$

Corollary 4.7. (i) The operators $\mathcal{D}_{k}$ and $\tilde{\mathcal{D}}_{k}$ have the same eigenvalues.

(ii) The spectrum of $\mathcal{D}_{k}$ and $\tilde{\mathcal{D}}_{k}$ is symmetric, i.e. if $\lambda$ is an eigenvalue, so is $-\lambda$.

(iii) The kernels of $\mathcal{D}_{k}$ and $\tilde{\mathcal{D}}_{k}$ are one-dimensional, if $(k+1) / 2$ is odd, and trivial otherwise.

Proof. One easily verifies that the characteristic polynomials of $\mathcal{D}_{k}$ and $\tilde{\mathcal{D}}_{k}$ are the same and of the form $\lambda p_{k}\left(\lambda^{2}\right)$, if $(k+1) / 2$ is odd, and $p_{k}\left(\lambda^{2}\right)$ otherwise, for some polynomial $p_{k}$. Since $a_{k, 1}, \ldots, a_{k,(k-1) / 2}$ are non-zero, the dimension of the kernel of $\mathcal{D}_{k}$ is at most 1 . Moreover, if $(k+1) / 2$ is even, then

$$
\operatorname{det}\left(\mathcal{D}_{k}\right)=\prod_{r=1}^{(k+1) / 4} a_{k, 2 r-1}^{2} .
$$

Corollary 4.8. The spectrum of the symplectic Dirac operators $\mathcal{D}$ and $\tilde{\mathcal{D}}$ on $\mathbb{C} P^{1}$ is unbounded above and below.

Proof. Let $\mathbf{U}_{k}$ be endowed with the norm defined by

$$
\left\|\sum_{l=0}^{(k-1) / 2} z_{l} L_{k, l}^{\circ}\right\|^{2}=\sum_{l=0}^{(k-1) / 2}\left|z_{l}\right|^{2}
$$

and let $\left\|\mathcal{D}_{k}\right\|$ be the corresponding operator norm of $\mathcal{D}_{k}$. Since the matrix representation of $\mathcal{D}_{k}$ with respect to $\left\{L_{k, 0}^{\circ}, \ldots, L_{k,(k-1) / 2}^{\circ}\right\}$ is Hermitian and the spectrum of $\mathcal{D}_{k}$ is symmetric, $\left\|\mathcal{D}_{k}\right\|$ is the absolut value of the largest and smallest eigenvalue of $\mathcal{D}_{k}$. Now the assertion follows from

$$
\left\|\mathcal{D}_{k}\right\| \geq\left\|\mathcal{D}_{k} L_{k, 0}^{\circ}\right\|=a_{k, 1} \geq \frac{k-1}{2} .
$$


Remark 4.9. The spectrum of the second order operator $\mathcal{P}$ on $\mathbb{C} P^{1}$ was already computed in [10]. For the general case, the coincidence of the spectra of $\mathcal{D}$ and $\tilde{\mathcal{D}}$ and their symmetry was proven in [11] (cf. also [12]) by means of a Fourier transform for symplectic spinor fields. For an explicit calculation of the eigenvalues and eigenvectors of $\mathcal{D}_{k}$ and $\tilde{\mathcal{D}}_{k}$ for small $k$, see [19].

Open Access This article is distributed under the terms of the Creative Commons Attribution Noncommercial License which permits any noncommercial use, distribution, and reproduction in any medium, provided the original author(s) and source are credited.

\section{References}

[1] Bär, C.: Das Spektrum von Dirac-Operatoren. Dissertation, Universität Bonn (1990)

[2] Bieliavsky, P.: Semisimple symplectic symmetric spaces. Geom. Dedicata 73, 245273 (1998)

[3] Borel, A., Wallach, N.: Continuous cohomology, discrete subgroups, and representations of reductive groups. Mathematical Surveys and Monographs 67. Providence: AMS (2000)

[4] Cahen, M., Gutt, S.: Spin structures on compact simply connected Riemannian symmetric spaces. Simon Stevin 62, 209-242 (1988)

[5] Cahen, M., Gutt, S., Rawnsley, J.: Symmetric symplectic spaces with Ricci-type curvature. Conférence Moshé Flato Quantization, deformations, and symmetries (Dijon, 1999). Math. Phys. Stud. 22, 81-91 (2000)

[6] Fricke, J., Habermann, L.: On the geometry of moduli spaces of symplectic structures. Manuscripta Math. 109, 405-417 (2002)

[7] Fricke, J., Habermann, K., Habermann, L.: On the existence of pseudo-Riemannian metrics on the moduli space of symplectic structures. Differ. Geom. Appl. 23, 17-25 (2005)

[8] Habermann, K.: The Dirac operator on symplectic spinors. Ann. Glob. Anal. Geom. 13, 155-168 (1995)

[9] Habermann, K.: Basic properties of symplectic Dirac operators. Commun. Math. Phys. 184, 629-652 (1997)

[10] Habermann, K.: Harmonic symplectic spinors on Riemann surfaces. Manuscr. Math. 94, 465-484 (1997)

[11] Habermann, K., Klein, A.: A Fourier transform for symplectic spinors and applications. Preprint, Universität Greifswald (2002)

[12] Habermann, K., Habermann, L.: Introduction to Symplectic Dirac Operators. Lecture Notes in Mathematics 1887. Springer, Berlin (2006)

[13] Habermann, K., Habermann, L., Rosenthal, P.: Symplectic Yang-Mills theory, Ricci tensor, and connections. Calc. Var. Partial Differ. Equ. 30, 137-152 (2007)

[14] Kadlčáková, L.: Dirac operator in contact symplectic parabolic geometry. Proceedings of the 21st Winter School "Geometry and Physics" (Srní, 2001). Rend. Circ. Mat. Palermo (2) Suppl. 69, 97-107 (2002)

[15] Kobayashi, S., Nomizu, K.: Foundations of differential geometry II. Interscience Publishers, New York (1969)

[16] Kostant, B.: Symplectic Spinors. Symp. math. 14, Geom. simplett., Fis. mat., Teor. geom. Integr. Var. minim., Convegni 1973, 139-152 (1974)

[17] Krýsl, S.: Classification of 1st order symplectic spinor operators over contact projective geometries. arXiv:0710.1425v1 [math.DG] 
[18] Loos, O.: Symmetric spaces I. W.A. Benjamin, Inc., New York (1969)

[19] Rudnick, S.: Symplektische Dirac-Operatoren auf symmetrischen Räumen. Universität Greifswald, Diplomarbeit (2005)

[20] Sommen, F.: An extension of Clifford analysis towards super-symmetry. Papers of the 5th International Conference Clifford Algebras and their Applications in Mathematical Physics (Ixtapa-Zihuatanejo, 1999). Prog. Phys. 19, 199-224 (2000)

[21] Tondeur, B.: Affine Zusammenhänge auf Mannigfaltigkeiten mit fastsymplektischer Struktur. Comment. Math. Helv. 13, 234-244 (1961)

[22] Wallach, N.R.: Harmonic analysis on homogeneous spaces. Pure and Applied Mathematics 19. Marcel Dekker, Inc., New York (1973)

[23] Wilson, P.M.H.: Some remarks on moduli of symplectic structures. Manuscripta Math. 116, 93-98 (2005)

[24] Wyss, C.: Symplektische Diracoperatoren auf dem komplexen projektiven Raum. Universität Bremen, Diplomarbeit (2003)

[25] Zelobenko, P.D.: Compact Lie groups and their representations. Translations of Mathematical Monographs 40. American Mathematical Society, Providence (1973) 